\title{
Seguimiento a resultados de evaluación del desempeño docente
}

Martha Patricia Hernández Beltrán mhernandezb@ittepic.edu.mx

\section{Claudia Lina Esmeralda Villa Arias}

cvillaa@ittepic.edu.mx

Sonia Espinoza Morales sespinoza@ittepic.edu.mx

Nidia Teresa Luna Vázquez nluna@ittepic.edu.mx

\section{Briseida Sandoval González}

bsandoval@ittepic.edu.mx

Tecnológico Nacional de México/Instituto Tecnológico de Tepic

Nayarit-México.

\section{RESUMEN}

Evaluar el desempeño docente es una actividad compleja que involucra distintos actores, en este trabajo se considera la perspectiva de los administradores del proceso de evaluación, para analizar la utilidad, seguimiento e impacto de los resultados de la evaluación docente desde su función en una escuela de nivel superior pública en México. Para lo anterior, se realizaron entrevistas a los jefes de departamento académico en el año 2020, mismos que fungen como administradores del proceso, y de quienes jerárquicamente depende todo el personal docente de la Institución, los datos recopilados permiten identificar si la evaluación cumple con su propósito de coadyuvar en la mejora continua, y describir la opinión que se tiene sobre la importancia, confiabilidad y validez del instrumento con que se realiza la evaluación docente. Los resultados que se publican corresponden a uno de los objetivos planteados dentro de una investigación más extensa que analiza también la opinión de los docentes acerca del instrumento de evaluación docente.

Palabras clave: evaluación; desempeño docente; docencia. 


\title{
Follow-up of the results of the evaluation of the teaching performance
}

\begin{abstract}
Evaluating teaching performance is a complex activity that involves different actors, this paper considers the perspective of the administrators of the evaluation process, to analyze the usefulness, monitoring and impact of the results of teacher evaluation from their role in a public high school in Mexico. For this purpose, interviews were conducted with the heads of academic departments in 2020, who serve as administrators of the process, and on whom hierarchically depend all the teaching staff of the institution. The data collected allow us to identify whether the evaluation fulfills its purpose of contributing to continuous improvement, and to describe the opinion held on its importance, reliability and validity of the instrument used for the teacher evaluation. The results published here correspond to one of the objectives of a more extensive investigation that also analyzes the opinion of teachers about the teacher evaluation instrument.
\end{abstract}

Key words: evaluation, teaching performance, teaching.

Artículo recibido: 02 noviembre. 2021 Aceptado para publicación: 28 noviembre 2021 Correspondencia: bsandoval@ittepic.edu.mx Conflictos de Interés: Ninguna que declarar 


\section{INTRODUCCIÓN}

Cada una de las instituciones de educación superior en México tiene un sin fin de actividades diversas que están vinculadas a las continuas evaluaciones, desde procesos de acreditación de carreras y certificaciones del proceso educativo por organismos externos donde se establecen requisitos e indicadores de cumplimiento y permiten hacer una autoevaluación con propósito de establecer la mejora contínua. De igual manera se plasma en los planes nacionales e institucionales o programas de desarrollo metas relacionadas con el mejoramiento de la calidad de la educación (Gobierno de México, 2019; TECNM, 2019; ITTEPIC, 2019).

Desde los años noventa la evaluación forma parte del plan de educación, esto ha evolucionado y se han generado políticas sustentadas en la evaluación dando lugar a los programas, procesos y acciones, estas políticas siguen vigentes y con el tiempo se han ido ajustando de acuerdo a cada Institución de Educación Superior.

Se observa que, en el contexto de la política educativa nacional y la calidad de los servicios ofrecidos por las Instituciones Educación Superior, un elemento clave es el papel desempeñado por los docentes, de ahí el interés por evaluar su actividad. Atender a la valoración de las actividades de los profesores se justifica por una de las finalidades de la evaluación son:

1. La orientada al mejoramiento de la enseñanza, a través de la retroalimentación proporcionada a los docentes

2. La actualización permanente y sus repercusiones.

Para Gómez López \& Valdés (2019) la evaluación del desempeño docente tiene un propósito formativo y se sustenta en la necesidad de conocer la práctica de los profesores en los escenarios educativos — aulas, talleres, laboratorios, espacios de práctica — para decidir qué aspectos deben reforzarse, mejorarse o modificarse en las dimensiones disciplinarias, pedagógicas, tecnológicas, comunicativas o éticas del ejercicio de los profesores.

En este mismo sentido, Martínez et al. (2016) refieren que la evaluación del desempeño docente dentro de aula, debe proporcionar un panorama real de lo que se está haciendo o se deja de hacer y ofrecer al docente una retroalimentación de su labor de acuerdo a los resultados de su evaluación; debido a que el instrumento por sí mismo no generará los cambios en la práctica profesional o ni las mejoras necesarias en ella. Es por ello que el 
enfoque de la evaluación debe ser formativa.

Peniche et al., (2020) a través de su estudio de enfoque cualitativo reconocen que los factores relativos al desempeño docente se clasifican en dos grupos: internos y externos. El primero de ellos corresponde a cuestiones propias del docente, en donde su implicación es directa: compromiso docente. Las categorías incluidas en la agrupación de factores externos, son aquellas que afectan al docente, sin que él tenga una injerencia directa, son elementos o circunstancias dadas por el entorno (la escuela o el sistema educativo): clima organizacional, dotación de materiales y recursos para la enseñanza, capacitación y actualización, estabilidad laboral y carga horaria.

Galaz et al. (2019) a través de un estudio comparativo mencionan que, de todos los modelos posibles de evaluación, en Chile y México se ha implementado el más conservador y el menos formativo; que se enfoca en los aspectos externos de la practica educativa, sin tomar en cuenta el contexto o características de la escuela. Dicho modelo está centrado en el rendimiento individual, con fines de medir clasificar; y que promueve más las consecuencias sancionadoras y menos a las acciones de reconocimiento y desarrollo profesional para los docentes. Argumentan que las evidencias corren más bien a favor de los países y sistemas evaluativos que confían y reconocen a sus profesoras y profesores, que generan condiciones laborales dignas, finalidades colectivas, reflexión y cooperación; aquéllos que anteponen la creatividad a la estandarización (Hargreaves y Shirley, 2007 citado por Galaz et al. 2019).

TantaleánOdar et al. (2016) a través de su investigación de tipo cuasi experimental, longitudinal y explicativa, confirman que el monitoreo pedagógico entendido como una estrategia de la supervisión, y que consiste en dar seguimiento permanente a las tareas asignadas al docente, con el objetivo de conocer el nivel de su desempeño para asesorarlo y capacitarlo según sus resultados; es un factor clave para que el docente eleve su nivel de desempeño. La supervisión realizada por el director o directivos docentes no debe limitarse a una simple inspección, sino que es necesario complementar el monitoreo con acciones de acompañamiento y asesoramiento pedagógico.

Concluyen que la atención de las deficiencias pedagógicas depende mucho del reconocimiento y aceptación de éstas, por lo que un estudio situacional correcto, permite fundamentar y convencer a los docentes de la existencia de sus aciertos y limitaciones pedagógicas (TantaleánOdar et al. 2016). 
Debido a su importancia y complejidad es importante analizar de manera integral el proceso de evaluación, considerar la opinión de sus usuarios, así como los beneficios que se obtienen al llevar a cabo este proceso. Como referencia en el Instituto Tecnológico de Tepic se han llevado a cabo estudios para analizar la opinión de los estudiantes (Sandoval et al., 2020) y de los docentes (Villa et al., 2021) en relación al instrumento de evaluación del desempeño docente vigente; lográndose identificar áreas de mejora como reducir el número de preguntas del cuestionario; revisar la pertinencia de las preguntas para asegurar que realmente nos ayuden a reconocer la calidad de los docentes y; demostrar por un lado a los estudiantes de que su opinión cuenta y sirve para algo, y para los docentes proporcionar la retroalimentación necesaria con base en los resultados de la evaluación como parte del seguimiento.

Derivado de esos estudios, se propuso realizar este trabajo de investigación; se plantearon los objetivos de analizar el impacto que se tiene del proceso de la evaluación docente en el Instituto Tecnológico de Tepic, describir la utilidad de los resultados del proceso de evaluación y las estrategias de seguimiento que se realizan desde la función de los jefes académicos o directivos docentes.

\section{MARCO TEÓRICO}

\section{La evaluación del desempeño}

Es importante considerar que "los sistemas efectivos de administración de la calidad son dinámicos; es decir, son capaces de adaptarse a los cambios para satisfacer las necesidades, requerimientos y expectativas de sus clientes" (Summers, 2006), pero no podrán llevarse a cabo si no existieran las herramientas adecuadas para la evaluación continua, como es el caso de la evaluación de desempeño docente, el cual es analizado en este proyecto, por su valioso aporte que debe tener para la capacitación y el desarrollo de los docentes, entendiendo el desarrollo como a la adquisición de capacidades, conocimientos, habilidades, actitudes y competencias que se obtienen a través de las actividades de la organización que inciden positivamente en la creación de nuevos conocimientos (Chiavenato, 2017).

Respecto al concepto de evaluación del desempeño Chiavenato (2014), lo define como "una apreciación sistemática de cómo cada persona se desempeña en un puesto y de su potencial de desarrollo futuro". Sin olvidar que, las acciones o comportamientos en los empleados son relevantes para los objetivos de la organización, y que pueden ser medidos 
en términos de las capacidades de cada individuo y su nivel de contribución a la empresa. Mientras que Martínez y Martínez consideran que la evaluación al desempeño debe servir para hacer un comparativo entre las conductas reales y las esperadas de los trabajadores, a través del uso de procedimientos e instrumentos adecuados y que en caso de resultados poco satisfactorios se puedan generar medidas correctivas como la capacitación y rediseño o cambio de puestos (Martínez y Martínez, 2009).

El proceso de evaluación del desempeño puede perseguir diferentes fines dentro de la organización, por tal motivo, cuando se diseña debe cuidarse que las dimensiones seleccionadas son pertinentes para los fines que persigue el proceso. La naturaleza y dinamismo de los puestos puede requerir de diferentes dimensiones para medir el desempeño de su ocupante, ya que dentro de las organizaciones existen puestos con muy diferentes niveles en contenido y en responsabilidad.

De acuerdo a Juárez (2018), los principales usos que las organizaciones dan a los resultados de la evaluación del desempeño son: la administración de la compensación, la evaluación se utiliza para despertar el interés y la ambición de los empleados por lograr mejores resultados a futuro; otro uso es para la detección de necesidades de capacitación y formación, ya que al evaluar el desempeño se está realizando un juicio del grado en que el empleado lograr los resultados en sus puestos que la organización requiere, es en la evaluación misma en la que el jefe inmediato tiene la oportunidad de identificar las razones que están limitando el desempeño de sus subordinados. Para la evaluación de los procesos de reclutamiento y selección ya que cuando una organización toma la decisión de contratar a una persona es porque ha elaborado un pronóstico de actuación de esa persona y se considera que cubre las características, capacidades, competencias y, en general, el potencial que se requiere para desempeñarse satisfactoriamente. $Y$ en la actualización de inventarios de capacidades y/o competencias humanas de la organización. El inventario de recursos humanos es un documento que contiene los datos relativos a los antecedentes personales y laborales, conocimientos especializados, capacidades humanas, competencias críticas, potencial laboral, intereses y necesidades personales, aspiraciones de carrera y la etapa de vida en la que se encuentran los empleados de la organización, principalmente. Lo anterior con la finalidad de predecir el desempeño en su puesto actual y en alguno otro de mayor responsabilidad en el futuro de la organización y sus aspiraciones y necesidades relacionadas con la etapa de su ciclo 
de vida laboral (Juárez, 2018).

\section{Responsabilidad por el proceso de evaluación del desempeño}

La operación de un sistema de evaluación del desempeño implica el involucramiento de diversos actores de la organización. Es decir, la planeación, el diseño, la evaluación misma, el manejo de los resultados, no puede ser responsabilidad de una sola persona. Cada una de las actividades del sistema tiene sus propias características, y exige no solo el conocimiento, sino también la experiencia en el manejo de la información.

El principal interesado en la evaluación del desempeño debiera ser el empleado, ya que la evaluación reduce la incertidumbre al proporcionarle retroalimentación de su desempeño, el empleado comprende lo que la organización espera de él, y busca la consonancia porque permite intercambiar ideas para lograr el entendimiento de conceptos entre el empleado y su superior.

Las organizaciones emplean distintas formas para determinar quién debe evaluar el desempeño de los empleados. Existen desde las más democráticas y participativas que dejan en manos del propio empleado la responsabilidad de autoevaluar libremente su desempeño. Algunas asignan la responsabilidad al jefe o superior inmediato, otras tratan de integrar al superior inmediato y al subordinado en la evaluación, y en la actualidad, se incluyen también la participación de externos en la evaluación.

Cada una de las organizaciones establece sus propias políticas en cuanto al sistema de evaluación del desempeño, y de acuerdo a esta política, definen a los responsables de la evaluación y de la administración del sistema.

Chiavenato (2008) menciona los diferentes actores y sus respectivas responsabilidades en la evaluación del desempeño. En primera instancia, el gerente que, como administrador de personas, tiene una responsabilidad de línea por el desempeño de sus subordinados, de su constante evaluación y de la comunicación de los resultados. El empleado o evaluado quien es responsable de evaluar su propio desempeño en función de la realimentación que le proporcione el gerente. Algunas organizaciones asignan la evaluación del desempeño a una comisión especialmente diseñada para tal efecto. Se refiere a una evaluación colectiva a cargo de un grupo de personas directa o indirectamente interesadas en el desempeño de los empleados.

\section{La evaluación del desempeño docente}

Evaluar el desempeño docente es un proceso por medio del cual se busca emitir juicios 
valorativos sobre el cumplimiento de las responsabilidades del docente en la enseñanza, aprendizaje y desarrollo de sus estudiantes a partir de información válida, objetiva y fiable relacionada con los logros de sus estudiantes y el desarrollo de sus áreas de trabajo (Tejedor, 2012).

Para Díaz (2017) no hay una mejora esencial en los procesos educativos, sea de aprendizaje, de trabajo docente o de procesos institucionales ya que la evaluación del desempeño docente se basa en un modelo de evaluación que segmenta e individualiza el acto de evaluación; derivado de sostenerse en una perspectiva gerencial. Expresa que se ha pasado de manera poco perceptible del reconocimiento de rasgos cualitativos al establecimiento de indicadores que cuantifican un desempeño académico, lo genera que sea la prioridad la mejora del indicador y no la mejora del proceso educativo en el contexto particular que se desarrolla.

Es por ello la importancia de la retroalimentación y seguimiento a los resultados cuantitativos. Para Tejedor (2012), la evaluación de los docentes no debe verse como una estrategia de vigilancia jerárquica que controla las actividades de los profesores, sino como una forma de fomentar y favorecer el perfeccionamiento del profesorado, como una manera de identificar las cualidades que conforman a un buen profesor para, a partir de ahí, generar políticas educativas que coadyuven a su generalización.

Valdés (2000), identifica varios de los fines que se persiguen con la evaluación del desempeño docente, entre los que menciona son los siguientes:

- El mejoramiento de la institución y de la enseñanza en el aula, ya que se busca un desarrollo educativo continuo, a lo largo del cual el personal docente de la institución puede mejorar. Cuando se integra eficazmente el desarrollo del personal, la evaluación del profesorado y la mejora de la institución se obtienen con una mayor eficacia.

- La responsabilidad y el desarrollo profesional, es decir aun cuando las responsabilidades son un elemento clave en el desempeño docente, la responsabilidad aquí se refiere fundamentalmente a la profesión y a sus estándares en la práctica y ética.

- El control administrativo considera la enseñanza como un aspecto clave de empleo, que requiere de la supervisión y el control del maestro por parte de la unidad administrativa. 
- En el pago por mérito, ya que se estima que los docentes necesitan del reconocimiento y de la motivación que proporcionan los incrementos salariales.

En México se reporta el uso de los cuestionarios de apreciación estudiantil como la estrategia privilegiada para evaluar el desempeño docente, el $98 \%$ de las instituciones encuestadas los utilizan, de ellas, $86 \%$ evalúa mediante este medio al total de la población de docentes y 12\% los usa para evaluar a una muestra de profesores (Rueda, et al, 2011 citado por Arámburo y Luna, 2013).

Para realizar una evaluación del desempeño docente es necesario establecer el perfil del buen docente en un contexto institucional, se deben definir los conocimientos, habilidades y actitudes que idealmente tendría que dominar un profesor, y con base en ellos se deben elaborar los instrumentos de evaluación del desempeño docente, el contexto de la institución como el modelo educativo vigente constituyen marcos de referencia distintos para cada organización escolar (Pacheco et al., 2018).

La construcción de instrumentos válidos sin duda constituye un proceso complejo en que se debe tomar en cuenta el contexto y a los usuarios del sistema de evaluación; ya sea docentes, directivos docentes y estudiantes que permita recopilar la información pertinente y concreta para ofrecer la oportunidad de retroalimentación y seguimiento al desempeño docente.

\section{La evaluación del desempeño docente en el Instituto Tecnológico de Tepic}

El Instituto Tecnológico de Tepic es un campus del Tecnológico Nacional de México, sistema que agrupa a más de 260 Institutos Tecnológicos en todo el país, el cual emana lineamientos academicos-administrativos a nivel central y coordina diferentes procesos relacionados con la docencia e investigación. De esta manera desde el 2013 se emitió de manera oficial el instrumento de evaluación del desempeño docente el cual está vigente a la fecha. El cual es contestado por los estudiantes aproximadamente un par de semanas antes de concluir el periodo de clases. Este instrumento identifica y reconoce en el docente un conjunto de competencias básicas que, al declararse, definen en forma explícita el desempeño del docente. Las competencias vigentes del perfil docente declaradas en el Cuestionario de Evaluación docente con enfoque en competencias (DGEST, 2013), son las siguientes: Disciplina, planeación del curso, diseño de ambientes de aprendizaje, estrategias, mètodos y técnicas de aprendizaje; motivación, evaluación del aprendizaje, comunicación, gestión del curso y por último la dimensión de tecnologías de la 
información y comunicación. El total de ítems de este instrumento es de 48 incluyendo una dimensión de satisfacción general y se aplica a través del sistema integral de información del Instituto.

\section{ESTRATEGIAS METODOLÓGICAS O MATERIALES Y MÉTODOS}

Esta investigación se efectuó bajo un enfoque de estudio de caso dentro del paradigma cualitativo de investigación, con el propósito de describir y comprender la forma en que se da seguimiento a los resultados de la evaluación del desempeño docente en un Instituto de educación superior público en México, para ello se realizaron 9 entrevistas bajo una guía de preguntas cerradas y abiertas a directivos docentes, quienes tienen a su cargo al personal académico de la Institución, aproximadamente 260 docentes; y también se entrevistó a la coordinadora el proceso de evaluación docente. Se revisaron así mismo documentos internos y procedimientos declarados para constar información proporcionada en las entrevistas (TECNM, 2019; ITTEPIC, 2019; ITTEPIC, s/f.).

Una vez realizadas las entrevistas se analizaron las respuestas bajo las siguientes categorías de análisis: Apreciación de la importancia de la evaluación, Utilidad de los resultados de evaluación, Seguimiento a resultados, Veracidad de los resultados obtenidos a través del CEDD. El levantamiento de datos se efectuó en el semestre enero-junio 2020 a través de medios virtuales. Con la información recabada se hizo un análisis que permitió dar respuesta a las preguntas planteadas.

\section{RESULTADOS Y DISCUSIÓN}

Se encontró que 6 de 9 jefes de departamento académico tienen de 1 a 5 años de antigüedad en el puesto, mientras que, para la categoría de más de 5 años de antigüedad, son solo 2 de 9 . Por otra parte, 1 jefe académico tiene entre 1 mes a 1 año de antigüedad al momento de recabar la información.

Tabla 1. Antigüedad de los administradores del proceso (jefes académicos).

\begin{tabular}{|rl|}
\hline \multicolumn{2}{|c|}{ Antigüedod } \\
\hline 1 mes a 1 año & 1 \\
1 año a 5 años & 6 \\
Más de 5 años & 2 \\
\hline Total & 9 \\
\hline
\end{tabular}

A continuación, se presenta de manera concreta la información obtenida de las preguntas cerradas para conocer la utilidad e impacto de los resultados de la evaluación del desempeño docente para los jefes académicos: 
- 8 de los 9 jefes académicos consideran importante el cuestionario de evaluación y uno considera con menor importancia.

- 5 de los 9 jefes académicos no han implementado estrategias con los resultados del CEDD. Los 4 que si lo han hecho les ha servido para detectar necesidades de capacitación docente.

- 7 de 9 jefes académicos manifiestan que han dado retroalimentación individual a los profesores sobre su resultado.

- 4 de 9 jefes académicos reportan como impacto a este seguimiento que los resultados de la evaluación de los docentes han mejorado en los semestres siguientes a quienes se proporcionó retroalimentación.

- 8 de los 9 jefes académicos perciben que los docentes les otorgan media o baja importancia a los resultados del cuestionario, ya que lo consideran poco confiable.

- 7 de 9 jefes académicos mencionan que no existe un procedimiento institucional para el seguimiento de resultados del CEDD.

En una de las preguntas abiertas se les solicitó emitir alguna propuesta o recomendación para mejorar el proceso de evaluación de los profesores, en la tabla 2 se concentran sus respuestas.

Tabla 2. Propuestas de los Jefes académicos para mejorar el proceso de evaluación docente.

\begin{tabular}{|c|l|}
\hline \multicolumn{2}{|c|}{ Propuestas } \\
\hline 1 & $\begin{array}{l}\text { Aplicar la encuesta en el tiempo adecuado, para así generar } \\
\text { acciones correctivas en el curso. }\end{array}$ \\
\hline 2 & $\begin{array}{l}\text { Actualizar el cuestionario a condiciones que prevalecen en estos } \\
\text { momentos. }\end{array}$ \\
\hline 3 & $\begin{array}{l}\text { Estandarizar el proceso para análisis e implementación de } \\
\text { acciones remediales }\end{array}$ \\
\hline 4 & $\begin{array}{l}\text { Concientizar a los alumnos de contestar de manera responsable } \\
\text { el cuestionario para que las estrategias a implementar partan de } \\
\text { una base real. }\end{array}$ \\
\hline 5 & $\begin{array}{l}\text { Rediseñar la rúbrica y definir fechas de aplicación inmediatos al } \\
\text { término de cada periodo. }\end{array}$ \\
\hline 7 & $\begin{array}{l}\text { Procurar hacer menos preguntas, para que la intención de los } \\
\text { alumnos sea contestar a conciencia y no solo por cumplir }\end{array}$ \\
\hline 8 & $\begin{array}{l}\text { Modificar el cuestionario para que sea más claro, menos cargado } \\
\text { de preguntas y en otro momento del semestre }\end{array}$ \\
\hline 9 & $\begin{array}{l}\text { Hacer más labor de conciencia en nuestros docentes de la } \\
\text { importancia de estos datos o resultados para aprovecharlos para } \\
\text { ofrecer una educación de mayor calidad a nuestros estudiantes. }\end{array}$ \\
\hline $\begin{array}{l}\text { Aplicar menos preguntas, ya que los estudiantes por ser muchas } \\
\text { tienden a dar la misma calificación sin dar importancia real al } \\
\text { desempeño del profesor. }\end{array}$ \\
\hline
\end{tabular}




\section{Como análisis a estos resultados se puede apreciar que}

- Se reconoce como un proceso de evaluación institucional.

- Se observa que no existe un procedimiento institucional de seguimiento del proceso.

- Que menos de la mitad de los administradores realiza un seguimiento de los resultados de la evaluación docente.

- Se reconoce que la mayoría de los administradores reconocen el proceso mediante el cual identifican fortalezas y oportunidades de mejora.

- La mayoría de los administradores han aplicado estrategias con base a su experiencia y han impactado en la mejora de los resultados.

En relación al impacto de los resultados se identificó el siguiente hallazgo: no se cuenta con un mecanismo para la medición de los resultados, también se observa que se aplican estrategias aisladas no estandarizadas, de acuerdo a la experiencia y al nivel de involucramiento en el puesto por parte de los jefes académicos; por lo que es importante poner énfasis en el desarrollo de ese mecanismo o procedimiento adecuado de seguimiento y control del proceso.

Por otro lado, se reconoce la necesidad de concientizar y capacitar al personal directivo docente respecto al manejo de la información y estrategias que debe llevar a cabo con los datos que obtiene, resultado del proceso de evaluación semestral que se realiza, así como para implementar estrategias de monitoreo (TantaleánOdar, 2016); el personal directivo que tiene en sus manos poder establecer estrategias para mejorar el proceso de enseñanza aprendizaje e identificar áreas de mejora, tiene relativamente poco tiempo en el puesto como administradores del proceso.

También se llevó a cabo una entrevista al coordinador del proceso de evaluación al desempeño docente que de manera institucional es el área administrativa que coordina el levantamiento de la encuesta a los estudiantes, concentra y entrega resultados a los jefes académicos para que se haga llegar el resultado al docente, en relación a esta entrevista se destacan los siguientes puntos:

- La evaluación es un instrumento válido para todos los Institutos del sistema nacional de tecnológicos, no es manipulable, es aplicable para todas las asignaturas y contiene las competencias docentes del sistema.

- Los resultados se dan a conocer al Director y Subdirector académico del Instituto, enseguida a los jefes académicos considerados administradores del proceso, por parte 
del coordinador se realiza un análisis de los resultados, es decir; por departamento y por profesor, de manera general se les dice a los jefes académicos sobre la consideración de los resultados en el proceso de capacitación, actualización profesional y docente.

- No existe seguimiento por parte del coordinador para la mejora del proceso, ni de los resultados.

- La perspectiva del coordinador es que el proceso es objetivo y funciona, que tiene posibilidades de mejora.

- Se realiza un informe de evaluación docente, el cual se entrega al representante de calidad del instituto, subdirección académica y jefes académicos, en el informe viene un análisis y resultados estadísticos de lo general a lo particular.

- Las acciones correctivas o de seguimiento a los resultados realizadas por los jefes académicos no son notificadas al coordinador del proceso de evaluación.

- El proceso de evaluación docente no forma parte del Sistema de Gestión de la Calidad; anteriormente bajo la norma anterior ISO 9001-2008 sí se tenía un procedimiento de evaluación docente declarado; se desconocen las razones por que no se incluyó en la actualización de la ISO9001-2015.

- Propone como mejora la actualización del cuestionario cada 5 años de acuerdo a las nuevas necesidades y que exista en la parte final del cuestionario un espacio abierto para que los estudiantes puedan expresar su sentir de la manera en que ellos decidan y pueden dar una aportación muy genuina a sus profesores, y con ello que los resultados de los profesores sean valorados para tomar decisiones más profundas de capacitación para una mejoría que se vea reflejada en el aula y un seguimiento para la asignación de las asignaturas.

- En general concibe que es un proceso de mucha ayuda, que observa que muchos profesores lo descalifican cuando no son favorecidos en sus resultados, pero manifiesta que se debe respetar la percepción de los estudiantes, porque sin duda alguna es su forma de expresar su reconocimiento al desempeño del profesor.

El proceso de evaluación del desempeño docente desde la perspectiva de los administradores/directivos se manifiesta como un proceso institucional formal que no tiene seguimiento, que no está establecido en los procesos de mejora, que sería recomendable incluirlo en el sistema de gestión de la calidad para asegurarse de que se 
cumpla con el proceso adecuadamente, actualmente de forma parcial en el procedimiento de capacitación docente se menciona a los resultado de evaluación docente como información diagnóstica o de entrada, pero no figura en este sistema de calidad con indicadores de alcance y con procedimiento de acciones preventivas o correctivas.

\section{CONCLUSIÓN O CONSIDERACIONES FINALES}

Se evalúa la utilidad de los resultados de la Evaluación del desempeño docente desde la perspectiva de los administradores del proceso o jefes académicos. Ellos, aunque reconocen la importancia de realizar evaluaciones al desempeño de manera sistemática en la institución, no hacen completamente uso de los resultados. Se aprecia que menos de la mitad han implementado acciones a partir de los resultados obtenidos, y esto de acuerdo a su experiencia o propio criterio ya que no existe procedimiento institucional por el cual se guíen, quedando a voluntad y según alcance de cada jefe la intervención y manera de retroalimentar a los docentes a cargo. Por otro lado, se reconoce la necesidad de contar con un programa de formación o inducción al puesto de quienes adquieren nuevas responsabilidades como jefes de departamento, para sensibilizar y concientizar sobre los objetivos de evaluación, y capacitación para el seguimiento y tratamiento de resultados. La evaluación del desempeño es un proceso gerencial clave para cualquier organización, y al mismo tiempo una herramienta de la gestión de recursos humanos, ya que suministra información valiosa para enfocar acciones pertinentes que impacten en la mejora del desempeño de los colaboradores, y por consecuencia en los resultados organizacionales, contribuye a que, tanto los empleados, como sus jefes o supervisores orienten sus conocimientos, su tiempo, sus esfuerzos, sus recursos y acciones hacia aquellos resultados clave que determinan los resultados que alcanza una organización cuando pretende lograr sus objetivos. En el contexto educativo, la finalidad de la evaluación del desempeño es ofrecer retroalimentación al docente, proponer estrategias y acompañamiento en la adquisición de nuevas destrezas técnicas o pedagógicas, pero sin duda como afirma TantaleánOdar et al. (2016) esto será posible en la medida de que se haga conciencia y se reconozcan las áreas de mejora, así como también de la habilitación de los directivos docentes para llevar a cabo este proceso.

Para concluir es importante mencionar que la finalidad de la evaluación no debe ser exhibir o demeritar el trabajo docente, si no que puede ser utilizada para motivar y recompensar cuando los resultados son positivos, pensando que la motivación extrínseca 
es un factor que siempre debe estar sobre la mesa como una estrategia que debe utilizarse para mejorar el desempeño de los docentes.

\section{Trabajo a futuro}

Esta investigación se realizó como un estudio de caso, sería interesante observar resultados en otros campos del Tecnológico Nacional de México para comparar la manera en que se da seguimiento a los resultados de la evaluación del desempeño docente, ya que es el mismo instrumento que se aplica en todos los Institutos que pertenecen al TECNM, y no existe una guía de actuación o de seguimiento a resultados.

\section{LISTA DE REFERENCIAS}

Arámburo Vizcarra, V. y Luna Serrano, E. (2013). La influencia de las características, profesor y del curso en los puntajes de evaluación docente. Revista mexicana de investigación educativa, 18(58), 949-968. Recuperado en 27 de noviembre de 2021, de http://www.scielo.org.mx/scielo.php?script=sci_arttext\&pid=S1405$66662013000300012 \& \operatorname{lng}=$ es\&tlng=es.

Chiavenato, I. (2008). Gestión del talento humano. Mc Graw Hill

Chiavenato, I. (2014). Introducción a la Teoría General de la Administración, 8a. México.

Chiavenato, I. (2017). Comportamiento Organizacional, 3 a.

Díaz-Barriga, A. (2017). De la evaluación individual a una evaluación social-integrada: La institución educativa, su unidad. Instituto de investigaciones sobre la Universidad y la Educación-Universidad Nacional Autónoma de México, ISBN: 978-607-02-9094-7. México, pp. 327-364.

Dirección General de Educación Superior Tecnológica (DGEST). (2013). Evaluación

Docente con enfoque en Competencias. Recuperado de: http://tecnm.mx/docencia /evaluaciondocente

Gobierno de México (2019). Plan Nacional de Desarrollo 2019-2024, Estados Unidos

Mexicanos, Presidencia de la República. Publicado en el DOF el 12 de julio de 2019. http://dof.gob.mx/nota_detalle.php?codigo=5565599\&fecha=12/07/2019

Instituto Tecnológico de Tepic (ITTEPIC). (2019). Programa de Desarrollo Institucional 2020-2024. https://tepic.tecnm.mx/doc/PDI2019-2014ITTEPIC.html

Instituto Tecnológico de Tepic (ITTEPIC). (s/f). Sistema de Gestión de Calidad ISO 9001-2015. https://www.tepic.tecnm.mx/sgc2/index.html

Juárez, O. (2014). Administración de la compensación, sueldos, salarios, incentivos y 
prestaciones. México. Grupo Editorial Patria, S. A. de C. V.

Galaz-Ruiz, A. Jiménez-Vásquez, M. y Díaz-Barriga, A. (2019). Evaluación del desempeño docente en Chile y México. Perfiles Educativos. Vol. XLI, núm. 163, IISUE-UNAM.

Gómez López, L. F., \& Valdés, M. G. (2019). La evaluación del desempeño docente en la educación superior. Propósitos y Representaciones, 7(2). https://doi.org/10.20511/pyr2019.v7n2.255

Juárez, O. (2014). Administración de la compensación, sueldos, salarios, incentivos y prestaciones. México. Grupo Editorial Patria, S. A. de C. V.

Martínez, F. y Martínez, E. (2009). Capacitación por competencias: principios y métodos. Martínez Chairez, G., Guevara Araiza, A. y Valles Ornelas, M. (2016). El desempeño docente y la calidad educativa. Revista Ra Ximhai. Vol. 12(6), pp. 123-134 https://www.redalyc.org/pdf/461/46148194007.pdf

Pacheco Cámara, M. L. del C., Ibarra Bocardo, I., Iñiguez Galindo, M. E., Lee García, H., \& Sánchez, C. V. (2018). The evaluation of teacher's performance in higher education. Revista Digital Universitaria, 19(6). https://doi.org/10.22201/codeic.16076079e.2018.v19n6.a2

Peniche Cetzal, R., Mac, C. R., Guzmán Ramírez, C., \& Mora Osuna, N. (2020). Factores que Afectan el Desempeño Docente en Centros de Alta y Baja Eficacia en México. REICE. Revista Iberoamericana Sobre Calidad, Eficacia Y Cambio En Educación, 18(2), 77-95. https://doi.org/10.15366/reice2020.18.2.004

Summers, D. (2006) Administración de la calidad. México.

Sandoval, G., Hernández, M. y Espinoza, M. (2020) Opinión de estudiantes de educación superior sobre un instrumento de evaluación del desempeño docente. Ponencia presentada en el XXIII Congreso internacional sobre innovaciones en docencia e investigación en ciencias económico administrativas. APCAM, septiembre 30 y octubre 1 y 2 .

TantaleánOdar, L.R., Vargas- Velásquez, M. y López-Regalado, O. (2016). El monitoreo pedagógico en el desempeño profesional docente. Revista DIM / Año 11 - No 33 - $\begin{array}{lllll}\text { Marzo } & 2016 & - & \text { ISSN: }\end{array}$ https://dialnet.unirioja.es/servlet/articulo?codigo $=5407957$

Tecnológico Nacional de México (TECNM). (2019). Programa de Desarrollo 
Institucional 2019-2020. https://www.tecnm.mx/?vista=Programa_Institucional Tejedor, F. (2012). Evaluación del desempeño docente. Revista Iberoamericana de Evaluación Educativa, 5(1e). http://www.rinace.net/riee/numeros/vol5num1_e/art24.pdf

Villa, A., Espinoza M. y Sandoval, G. (2021). La Evaluación del Desempeño Docente desde la perspectiva del docente y de los administradores del proceso. Revista de Investigación Académica sin Frontera. Año 14 (Num. 35), ISSN 2007-8870. 